Research Paper

\title{
Diagnostic Performance of Diffusion MRI for differentiating Benign and Malignant Nonfatty Musculoskeletal Soft Tissue Tumors: A Systematic Review and Meta-analysis
}

\section{Qian Wang1\#, Xinguang Xiao"\#, Yanchang Liang' ${ }^{3}$, Hao Wen ${ }^{4}$, Xiaopeng Wen ${ }^{5}$, Meilan Gu ${ }^{1}$, Cuiping Ren ${ }^{6}$, Kunbin} $\mathrm{Li}^{4}$, Liangwen $\mathrm{Yu}^{3}$, Liming $\mathrm{Lu}^{2} \bowtie$

1. Department of Medical Imaging, Zhengzhou Central Hospital Affiliated to Zhengzhou University, 195 Tongbai Road, 455007, Zhengzhou, China.

2. Clinical Research and Data Center, South China Research Center for Acupuncture and Moxibustion, Medical College of Acu-Moxi and Rehabilitation, Guangzhou University of Chinese Medicine, Guangzhou, China.

3. Guangzhou University of Chinese Medicine, 510006, Guangzhou, China.

4. Department of Neurology, Sun Yat-sen Memorial Hospital, Sun Yat-sen University, 107 Yanjiang West Road, Guangzhou, China.

5. Department of neurological rehabilitation, Zhengzhou Central Hospital Affiliated to Zhengzhou University, 450000, Zhengzhou, China.

6. Department of Medical Imaging, The First Affiliated Hospital of Zhengzhou University, Zhengzhou, China.

\#Co-first authors with equal contributions to this work.

$\triangle$ Corresponding author: Clinical Research and Data Center, South China Research Center for Acupuncture and Moxibustion, Medical College of Acu-Moxi and Rehabilitation, Guangzhou University of Chinese Medicine, Guangzhou, China. E-mail: lulimingleon@126.com.

(C) The author(s). This is an open access article distributed under the terms of the Creative Commons Attribution License (https://creativecommons.org/licenses/by/4.0/). See http://ivyspring.com/terms for full terms and conditions.

Received: 2021.04.28; Accepted: 2021.10.02; Published: 2021.10.28

\begin{abstract}
Objective: To evaluate the diagnostic performance of standard diffusion-weighted imaging (DWI), intravoxel incoherent motion (IVIM), and diffusion kurtosis imaging (DKI), for differentiating benign and malignant soft tissue tumors (STTs).

Materials and methods: A thorough search was carried out to identify suitable studies published up to September 2020. The quality of the studies involved was evaluated using Quality Assessment of Diagnostic Accuracy Studies-2 (QUADAS-2). The pooled sensitivity (SEN), specificity (SPE), and summary receiver operating characteristic (SROC) curve were calculated using bivariate mixed effects models. A subgroup analysis was also performed to explore the heterogeneity.

Results: Eighteen studies investigating 1319 patients with musculoskeletal STTs (malignant, $n=623$; benign, $n=696$ ) were enrolled. Thirteen standard DWI studies using the apparent diffusion coefficient (ADC) showed that the pooled SEN and SPE of ADC were 0.80 (95\% Cl: 0.77-0.82) and 0.63 (95\% Cl: $0.60-0.67)$, respectively. The area under the curve (AUC) calculated from the SROC curve was 0.806 . The subgroup analysis indicated that the percentage of myxoid malignant tumors, magnet strength, study design, and ROI placement were significant factors affecting heterogeneity. Four IVIM studies showed that the AUCs calculated from the SROC curves of the parameters ADC and D were 0.859 and 0.874 , respectively. The AUCs for the IVIM parameters pseudo diffusion coefficient (D*) and perfusion fraction ( $f$ ) calculated from the SROC curve were 0.736 and 0.573 , respectively. Two DKI studies showed that the AUCs of the DKI parameter mean kurtosis (MK) were 0.97 and 0.89 , respectively.

Conclusion: The DWI-derived ADC value and the IVIM DWI-derived D value might be accurate tools for discriminating musculoskeletal STTs, especially for non-myxoid SSTs, using more than two b values, with maximal b value ranging from 600 to $800 \mathrm{~s} / \mathrm{mm}^{2}$, additionally, a high-field strength $(3.0 \mathrm{~T})$ optimizes the diagnostic performance.
\end{abstract}

Key words: diffusion weighted imaging; musculoskeletal; soft tissue tumors; meta-analysis

\section{Introduction}

Soft tissue tumors (STTs) are a very heterogeneous group of tumors with various presentations, and still pose an important challenge in daily clinical practice [1, 2]. Magnetic resonance imaging (MRI) is frequently used to characterize and grade soft tissue tumors. Some benign STTs, such as haemangioma and lipoma, have characteristic imaging features that can be correctly diagnosed with 
conventional MRI. However, for a large number of STTs with a nonspecific imaging appearance, conventional MRI is often not reliable for distinguishing malignant from benign soft tissue tumors [3-5].

In recent years, some studies have reported the usefulness of diffusion-weighted imaging (DWI) for benign and malignant STTs. DWI is a technique that is useful for qualitative and quantitative assessments of the motion of water in the tissue microenvironment [6-8]. The apparent diffusion coefficient (ADC) is a numerical parameter that is calculated from DWI. According to some investigators, malignant STTs usually have lower ADC values due to increased tumor cell packing $[9,10]$, and the ADC values for myxoid tumors were significantly higher than those of non-myxoid tumors [6, 10]. Other investigators reported overlapping ADC values for benign and malignant STTs [11]. However, ADC can be influenced not only by molecular diffusion restriction but also by capillary perfusion effects, therefore, ADC might have a limitation in characterizing the lesion $[12,13]$. Currently, intravoxel incoherent motion (IVIM) DWI has proposed by Le Bihan [14], more accurately separates molecular diffusion and capillary perfusion effects in each voxel and therefore encompasses both the capillary perfusion-related parameter pseudo diffusion coefficient $\left(\mathrm{D}^{*}\right)$, perfusion fraction (f), and the true molecular diffusion coefficient (D) $[12,13,15]$. Although several studies have demonstrated the use of IVIM DWI for the differentiation benign and malignant tumors of pancreas, liver, and breast [16-19], little information is available regarding the usefulness of IVIM parameters for evaluation of musculoskeletal STTs.

Conventional DWI techniques always assume a Gaussian diffusion in which water molecules diffuse without any restriction. However, due to the complexity of complex biological tissues, the diffusion of water molecules tends to deviate from a Gaussian distribution, thereby limiting the effectiveness of conventional DWI. Diffusion kurtosis imaging (DKI) is an advanced non-Gaussian diffusion imaging technique that has been used to account for this deficiency and is more suitable for assessments of the tumor microenvironment [20-22]. Previous studies have introduced the feasibility of DKI in brain, rectal, prostate, and breast tumors. However, few DKI studies have examined musculoskeletal STTs [20].

Previous studies were inconclusive because of insufficient samples, different histological tumor types and the use of different diagnostic algorithms, including $b$ values, cut-off values, and MRI parameters $[12,13,15,23]$. We conducted a systematic review and meta-analysis to provide an overview of the diagnostic performance of standard DWI, IVIM DWI, and DKI, for differentiating benign and malignant musculoskeletal STTs.

\section{Methods}

\section{Search strategy}

We performed this meta-analysis according to the Preferred Reporting Items for Systematic Reviews-Diagnostic Test Accuracy (PRISMA-DTA) guidelines [24]. A search of PubMed, CENTRAL (Cochrane Library), Embase, PMC, China National Knowledge Infrastructure (CNKI) and WANFANG databases from their inception until September 2020 was performed to find potentially qualified studies. Medical Subject Heading (MeSH) terms, keywords, or words appearing were used as the search strategy (S1). We did not impose language restrictions.

\section{Inclusion Criteria and Study Selection}

\section{Type of studies}

The inclusion criteria were (1) cohort studies that included retrospective or prospective studies; (2) the sensitivity (SEN) and specificity (SPE) of DWI for distinguishing benign and malignant STTs were reported clearly in the study; and (3) sufficient information was available for calculating true-positive (TP), false-positive (FP), false-negative (FN), and true-negative (TN) values for the statistical analysis.

The exclusion criteria were (1) studies that were reviews, case reports or case series, abstracts, letters, comments, editorials, or animal studies and (2) studies that had insufficient data for reconstructing $2 \times 2$ tables.

\section{Type of patients}

Patients with known musculoskeletal STTs who were referred for the initial lesion evaluation and had no history of treatment were included, all studies confirmed by histopathology, and excluded lipomatous tumors or vascular tumors.

\section{Type of imaging technique}

All studies used DWI, and ADC, DTI, DKI or IVIM parameters were calculated as the index test for differentiating benign and malignant STTs. The studies used other imaging techniques or used the DWI technique but did not pertain to the field of musculoskeletal STTs were excluded.

\section{Data Extraction}

A data extraction spreadsheet was developed to collect all related information, then we summarized the information in table of baseline characteristics, imaging characteristics and diagnostic results. 


\section{Diagnostic results}

The key information we extracted was the numbers of TP, FP, FN and TN using the DWI-derived parameter ADC; the IVIM DWI-derived parameters $\mathrm{D}, \mathrm{D}^{*}$, and $\mathrm{f}$; and the DKI-derived parameter mean kurtosis (MK) compared with histopathology. If TP, FP, TN, and FN were not reported, we calculated these values backwards using the indexes SEN, SPE, and number of malignant and benign lesions, and the results were rounded to the nearest whole number. For studies that reported multiple SEN and SPE due to the use of different combinations of $\mathrm{b}$ values, cut-off values, tumor pathologies, or different ROI placements, we extracted all the results. When studies included DWI and other MRI techniques, we only extracted the DWI data. When a study reported multiple histological classifications of STTs, we only extracted the data for benign and malignant tumors, as well as all soft tissue tumors (all STTs) and non-myxoid soft tissue tumors (non-myxoid STTs). When studies reported different ADC measurements, we only extracted the data for the minimal $\mathrm{ADC}$ value $\left(\mathrm{ADC}_{\min }\right)$ and mean $\mathrm{ADC}$ value $\left(A D C_{\text {mean }}\right)$. When studies had two readers, we extracted the reader with higher accuracy.

Other diagnostic results included the cut-off values, area under the curve (AUC), mean ADC, D, $\mathrm{D}^{*}, \mathrm{f}$, and MK for benign and malignant STTs.

\section{Basic study information}

We extracted the following information: first author, date of publication, patient age, sex, number of patients, number of benign and malignant STTs, type of tumors (STTs or non-myxoid STTs), reference standard and type of study design (prospective or retrospective).

\section{Basic Imaging information}

We extracted the following information: (1) imaging parameters used for DWI (magnetic field strength, MRI machine and vendor, coil used, b values, slice thickness, sequence type, repetition time/echo time (TR/TE), matrix size, and field of view (FOV)); (2) ADC measurements $\left(\mathrm{ADC}_{\text {mean }}\right.$ and $\left.\mathrm{ADC}_{\mathrm{min}}\right)$; and (3) region of interest (ROI) placement (manually over the solid portion or other).

\section{Data Quality Assessment}

Two reviewers independently assessed each study for quality and potential bias using the QUADAS-2 (Quality Assessment of Diagnostic Accuracy Studies, revised version) [25], which mainly consisted of 4 domains: (1) patient selection, (2) index test, (3) reference standard, and (4) flow and timing. When 5 or more than 5 terms were regarded as low risk, the study was classified as high quality. More than $70 \%$ of all included studies were high quality, and the quality of all included studies was regarded as high. Disagreements were reviewed in detail and subsequently settled by consensus.

\section{Statistical analysis}

First, patient demographic characteristics and extracted covariates were summarized using standard descriptive statistics. Continuous variables are presented as the means and 95\% confidence intervals (CIs), whereas categorical variables are presented as frequencies or percentages.

Second, the statistical analyses were performed using Meta-Disc 1.4 software (Ramón y Cajal Hospital, Madrid, Spain). Meta-analyses of standard DWI and IVIM DWI were performed as described below. First, heterogeneity between the included studies was evaluated. We evaluated the threshold effect by calculating the Spearman correlation coefficient between the logit of SEN and the logit of (1- SPE). The threshold effect was confirmed if the coefficient was $>0.6$ [26]. Apart from variations due to the threshold effect, heterogeneity might be generated from other related factors. Then, heterogeneity was determined using the Cochran $Q$ test $(P<0.05$ indicating the presence of heterogeneity) and the $I^{2}$ test (with $I^{2} \leq 25 \%$ indicating low heterogeneity, $25 \%<I^{2} \leq 50 \%$ indicating moderate heterogeneity, and $I^{2}>50 \%$ indicating significant heterogeneity) $[26,27]$. The SEN, and SPE of the included studies were pooled. According to the Cochrane review guidelines, if significant heterogeneity was present at $I^{2}>50 \%$, random-effects models were chosen; otherwise, fixed-effects models were used. We also calculated the AUC of the summary receiver operating characteristic curve (SROC). In general, a diagnostic tool is regarded as failed when AUC values range from 0.5 to 0.6 , poor when AUC values range from 0.6 to 0.7 , fair when AUC values range from 0.7 to 0.8 , good when AUC values range from 0.8 to 0.9 , and excellent when AUC values range from 0.9 to $1[26,27]$.

Third, if significant heterogeneity was detected, we then performed meta-regression analyses, setting $P<0.05$ to indicate a significant contribution to heterogeneity. Subgroups based on (1) ADC measurements $\left(\mathrm{ADC}_{\text {mean }}\right.$ versus the $\left.\mathrm{ADC}_{\text {min }}\right)$; (2) the number of $\mathrm{b}$ values ( $=2$ versus $>2)$; (3) maximal $\mathrm{b}$ value $\left(b_{\max } \leq 600 \mathrm{~s} / \mathrm{mm}^{2}\right.$ versus $600 \mathrm{~s} / \mathrm{mm}^{2}<b_{\max } \leq 800$ $\mathrm{s} / \mathrm{mm}^{2}$ versus $\mathrm{b}_{\max }>800 \mathrm{~s} / \mathrm{mm}^{2}$ ); (4) magnet strength (3.0 T versus $1.5 \mathrm{~T}$ ); (5) study design (prospective versus retrospective); (6) total number of patients $(>60$ versus 560 ); (7) ROI placement (manually over solid portion versus other); (8) tumor pathology (all soft tissue tumors versus non-myxoid soft tissue tumors); 
and (9) percentage of myxoid malignant tumors $(>10 \%$ versus $\leq 10 \%$ ) were analysed.

Last, due to the small sample size of the DKI studies, we did not perform a statistical analysis of the two studies and instead summarized their basic characteristics.

\section{Results}

\section{Literature research}

Figure 1 shows a flow diagram summarizing the literature search process. Ultimately, 18 studies $[9,15$, $20,23,28-41]$ that met the eligibility criteria were included in our meta-analysis (13 studies evaluated standard DWI, 3 studies evaluated IVIM DWI, 1 study evaluated both IVIM and DKI, and 1 study evaluated
DKI).

\section{Study characteristics}

\section{Patient characteristic}

Ultimately, 18 studies examining 1319 patients with musculoskeletal STTs (malignant, $n=623$; benign, $n=696)$ were included in this meta-analysis, which excluded lipomatous tumors and vascular tumors. Table 1 provides an overview of the patient characteristics in the 18 eligible studies. We obtained 27 diagnostic results of standard DWI, $6(22.2 \%)$ diagnostic results of IVIM DWI, and 2 (7.4\%) diagnostic results of DKI for malignant and benign STTs.

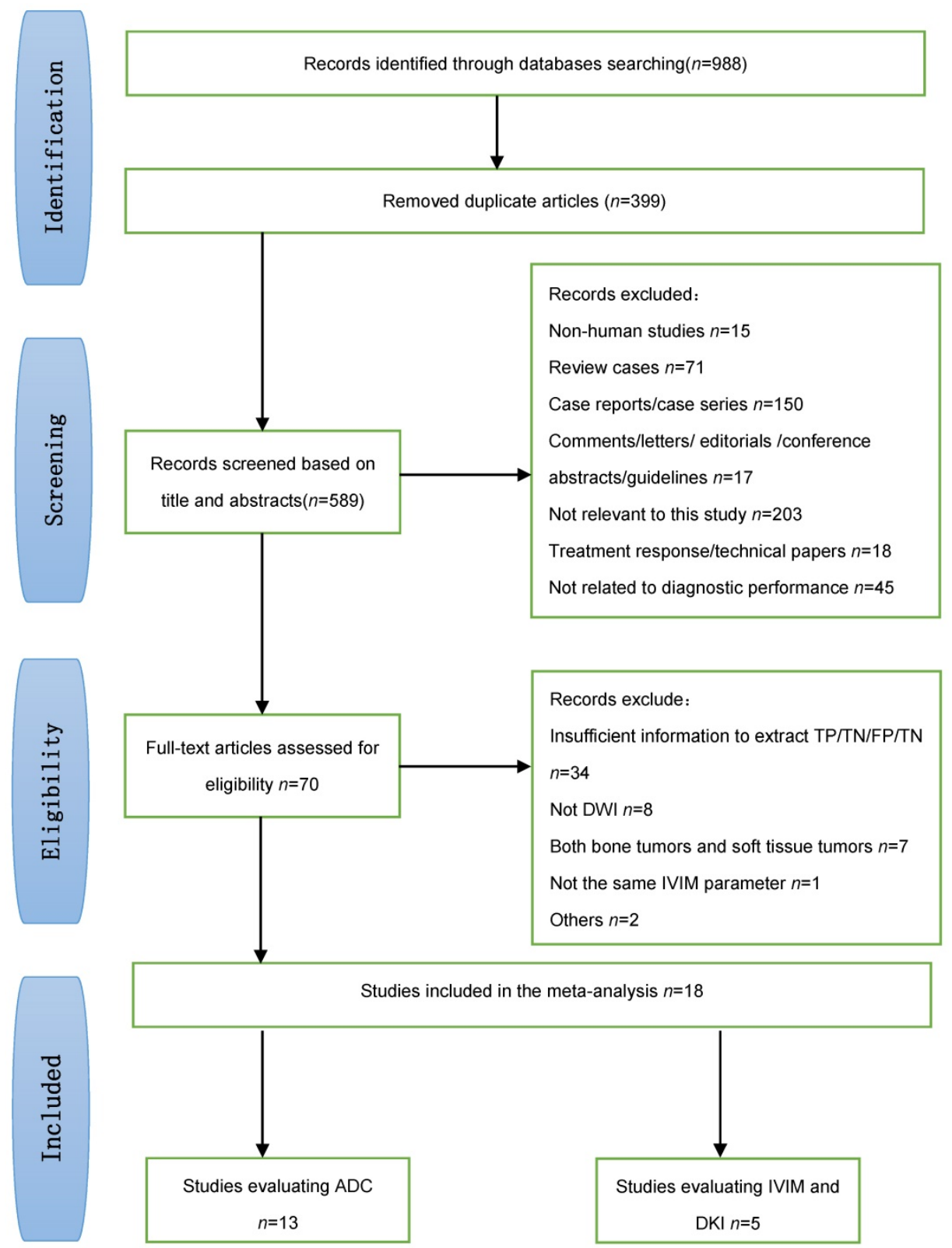

Figure 1. Flow diagram showing the study selection process for the meta-analysis. 
Table 1. Baseline characteristics of included studies

\begin{tabular}{|c|c|c|c|c|c|c|c|c|c|c|c|}
\hline $\begin{array}{l}\text { Author } \\
\text { year }\end{array}$ & County & $\begin{array}{l}\text { Study } \\
\text { type }\end{array}$ & $\begin{array}{l}\text { NO. } \\
\text { Patients } \\
(\mathrm{M} / \mathrm{F})\end{array}$ & $\begin{array}{l}\text { Age (years)mean } \\
\pm \mathrm{SD} \text { (range) }\end{array}$ & $\begin{array}{l}\text { NO. } \\
\text { Tumors } \\
(\mathrm{M} / \mathrm{B})\end{array}$ & $\begin{array}{l}\text { Pathology of } \\
\text { tumor }\end{array}$ & $\begin{array}{l}\text { Percentage } \\
\text { of myxoid } \\
\text { MTTs (\%) }\end{array}$ & $\begin{array}{l}\text { Reference } \\
\text { standard }\end{array}$ & MRI Technical* $^{*}$ & ROI placement & $\begin{array}{l}\text { Diffusion } \\
\text { parameters } \\
\text { evaluated }\end{array}$ \\
\hline $\begin{array}{l}\text { Lee SY (32) } \\
2016\end{array}$ & Korea & Retro & $63(35 / 28)$ & $51(17-90)$ & $34 / 29$ & All STTs & $20.3(5 / 34)$ & $\mathrm{HP}$ & $\begin{array}{l}\text { conventional } \\
\text { MRI } \\
\text { DWI }\end{array}$ & $\begin{array}{l}\text { manually/solid } \\
\text { tumor area }\end{array}$ & $\begin{array}{l}\mathrm{ADC}_{\text {min }} \\
\mathrm{ADC}_{\text {mean }}\end{array}$ \\
\hline $\begin{array}{l}\text { Del Grande F } \\
\text { (36) } 2016\end{array}$ & USA & Pros & $39(20 / 19)$ & $\begin{array}{l}\text { malignant } \\
53.7 \pm 21.5(21-82) \\
\text { benign } \\
44.6 \pm 21.1(1-87)\end{array}$ & $12 / 27$ & All STTs & $8.3(1 / 12)$ & $\begin{array}{l}\text { HP+follow } \\
\text { up }\end{array}$ & $\begin{array}{l}\text { conventional } \\
\text { MRI } \\
\text { DWI } \\
\text { static CE-MR } \\
\text { dynamic CE-MR }\end{array}$ & $\begin{array}{l}\text { manually/largest } \\
\text { area of tumor }\end{array}$ & $\begin{array}{l}\mathrm{ADC}_{\text {min }} \\
\mathrm{ADC}_{\text {mean }}\end{array}$ \\
\hline $\begin{array}{l}\text { Bonarelli C } \\
\text { (33) } \\
2014\end{array}$ & France. & Pros & $65(35 / 30)$ & $50(19-86)$ & $24 / 41$ & All STTs & $25(6 / 24)$ & $\mathrm{HP}$ & $\begin{array}{l}\text { conventional } \\
\text { MRI } \\
\text { DWI }\end{array}$ & $\begin{array}{l}\text { Manually/semi- } \\
\text { automatic/Cystic } \\
\text { solid, mixed }\end{array}$ & $\begin{array}{l}\mathrm{ADC}_{\text {min }} \\
\mathrm{ADC}_{\text {mean }}\end{array}$ \\
\hline $\begin{array}{l}\text { Dodin G (34) } \\
2020\end{array}$ & France & Pros & $\begin{array}{l}288 \\
(148 / 140)\end{array}$ & $50 \pm 17(18-90)$ & $104 / 184$ & non-myxoid STTs & 0 & $\begin{array}{l}\mathrm{HP}+\text { follow } \\
\text { up }\end{array}$ & $\begin{array}{l}\text { conventional } \\
\text { MRI } \\
\text { DWI/DCE/MRS }\end{array}$ & $\begin{array}{l}\text { manually/solid } \\
\text { tumor area }\end{array}$ & $\mathrm{ADC}_{\min }$ \\
\hline $\begin{array}{l}\text { Hassanien } \\
\text { OA (35) } 2018\end{array}$ & Egypt & Pros & $45(13 / 32)$ & $42 \pm 18.5(9-72)$ & $21 / 24$ & All STTs & $9.5(2 / 21)$ & $\begin{array}{l}\mathrm{HP}+\text { follow } \\
\text { up }\end{array}$ & $\begin{array}{l}\text { conventional } \\
\text { MRI } \\
\text { DWI }\end{array}$ & $\begin{array}{l}\text { manually/fleshy } \\
\text { tumor area }\end{array}$ & $\mathrm{ADC}_{\text {mean }}$ \\
\hline $\begin{array}{l}\text { Romeih M (9) } \\
2018\end{array}$ & Egypt & Pros & 50 & $33(1.5-75)$ & $28 / 22$ & All STTs & $14.3(4 / 28)$ & $\mathrm{HP}$ & $\begin{array}{l}\text { conventional } \\
\text { MRI } \\
\text { DWI }\end{array}$ & NA & $\mathrm{ADC}_{\text {mean }}$ \\
\hline $\begin{array}{l}\text { Teixeira PAG } \\
(37) \\
2016\end{array}$ & France & Pros & 76 & NA & $30 / 46$ & All STTs & $30(9 / 30)$ & $\mathrm{HP}$ & $\begin{array}{l}\text { conventional } \\
\text { MRI } \\
\text { DWI }\end{array}$ & $\begin{array}{l}\text { manually/solid } \\
\text { tumor area }\end{array}$ & $\begin{array}{l}\mathrm{ADC}_{\text {min }} \\
\mathrm{ADC}_{\text {ratio }}\end{array}$ \\
\hline $\begin{array}{l}\text { Song Y } \\
\text { (38) } 2017\end{array}$ & Korea & Retro & 51 & $46.2(7-81)$ & $31 / 20$ & non-myxoid STTs & 0 & $\mathrm{HP}$ & $\begin{array}{l}\text { conventional } \\
\text { MRI } \\
\text { DWI }\end{array}$ & $\begin{array}{l}\text { manually/solid } \\
\text { tumor area }\end{array}$ & $\begin{array}{l}\mathrm{ADC}_{\text {min }} \\
\mathrm{ADC}_{\text {mean }}\end{array}$ \\
\hline $\begin{array}{l}\text { Razek A } \\
\text { (28) } 2010\end{array}$ & Egypt & Retro & $37(22 / 15)$ & $41(4-68)$ & $23 / 14$ & non-myxoid STTs & 0 & $\mathrm{HP}$ & $\begin{array}{l}\text { conventional } \\
\text { MRI } \\
\text { DWI }\end{array}$ & $\begin{array}{l}\text { manually/solid } \\
\text { tumor area }\end{array}$ & $\mathrm{ADC}_{\text {mean }}$ \\
\hline $\begin{array}{l}\text { Lee JH } \\
\text { (29) } 2020\end{array}$ & Korea & Retro & $95(49 / 46)$ & $45.6(12-80)$ & $29 / 66$ & $\begin{array}{l}\text { All } \\
\text { STTs/non-myxoid } \\
\text { STTs }\end{array}$ & $27.6(8 / 29)$ & $\begin{array}{l}\mathrm{HP}+\text { follow } \\
\text { up }\end{array}$ & $\begin{array}{l}\text { conventional } \\
\text { MRI } \\
\text { DWI }\end{array}$ & $\begin{array}{l}\text { manually/solid } \\
\text { tumor area }\end{array}$ & $\mathrm{ADC}_{\text {mean }}$ \\
\hline $\begin{array}{l}\text { Robba T (30) } \\
2017\end{array}$ & Italy & Retro & $46(27 / 19)$ & $57(12-85)$ & $34 / 10$ & All STTs & $29.4(10 / 34)$ & $\begin{array}{l}\mathrm{HP}+\text { follow } \\
\text { up }\end{array}$ & $\begin{array}{l}\text { conventional } \\
\text { MRI } \\
\text { DWI }\end{array}$ & $\begin{array}{l}\text { manually/solid } \\
\text { tumor area }\end{array}$ & $\mathrm{ADC}_{\text {mean }}$ \\
\hline $\begin{array}{l}\text { Choi YJ (31) } \\
2018\end{array}$ & Korea & Retro & $136(68 / 68)$ & $57.2(16-86)$ & $63 / 73$ & All STTs & $5.5(4 / 73)$ & $\mathrm{HP}$ & $\begin{array}{l}\text { conventional } \\
\text { MRI } \\
\text { DWI } \\
\text { DCE }\end{array}$ & $\begin{array}{l}\text { manually/solid } \\
\text { tumor area }\end{array}$ & $\begin{array}{l}\mathrm{ADC}_{\text {min }} \\
\mathrm{ADC}_{\text {mean }} \\
\mathrm{ADC}_{\text {max }}\end{array}$ \\
\hline $\begin{array}{l}\text { Jeon JY (39) } \\
2016\end{array}$ & $\begin{array}{l}\text { South } \\
\text { Korea }\end{array}$ & Retro & $60(30 / 30)$ & $48.5(5-80)$ & $35 / 25$ & All STTs & $4.0(1 / 25)$ & $\mathrm{HP}$ & $\begin{array}{l}\text { conventional } \\
\text { MRI } \\
\text { DWI }\end{array}$ & $\begin{array}{l}\text { manually/solid } \\
+ \text { entire tumor } \\
\text { area }\end{array}$ & $\mathrm{ADC}_{\text {mean }}$ \\
\hline Wu H (15) 2017 & China & Pros & $65(35 / 39)$ & $48.4(12-75)$ & $47 / 23$ & All STTs & $8.7(2 / 23)$ & $\mathrm{HP}$ & $\begin{array}{l}\text { conventional } \\
\text { MRI } \\
\text { IVIM-DWI }\end{array}$ & $\begin{array}{l}\text { manually/solid } \\
\text { tumor area }\end{array}$ & $\begin{array}{l}\text { ADCstd, D, D* } \\
f\end{array}$ \\
\hline $\begin{array}{l}\text { Teixeira } \\
\text { PAG(23) }^{(23)} \\
2019\end{array}$ & France. & Pros & $64(23 / 41)$ & $52 \pm 17(18-92)$ & $35 / 29$ & $\begin{array}{l}\text { All } \\
\text { STTs/non-myxoid } \\
\text { STTs }\end{array}$ & $6.25(4 / 64)$ & $\mathrm{HP}$ & $\begin{array}{l}\text { conventional } \\
\text { MRI } \\
\text { IVIM-DWI }\end{array}$ & $\begin{array}{l}\text { manually/solid } \\
\text { tumor } \\
\text { area/entire area }\end{array}$ & $\begin{array}{l}\text { ADCstd, D, D* } \\
f\end{array}$ \\
\hline $\begin{array}{l}\text { Lee SK }{ }^{(40)} \\
2020\end{array}$ & Korea & Retro & $67(30 / 37)$ & $55 \pm 15(18-82)$ & $35 / 32$ & $\begin{array}{l}\text { All } \\
\text { STTs/non-myxoid } \\
\text { STTs }\end{array}$ & $18.7(6 / 32)$ & $\mathrm{HP}$ & $\begin{array}{l}\text { conventional } \\
\text { MRI } \\
\text { IVIM-DWI } \\
\text { DCE }\end{array}$ & $\begin{array}{l}\text { manually/solid } \\
\text { tumor area }\end{array}$ & $\begin{array}{l}\text { ADCstd, D, D* } \\
f\end{array}$ \\
\hline $\begin{array}{l}\text { Zhang X } \\
\text { (41)2018 }\end{array}$ & China & Pros & 26 & $45.3 \pm 19.9(6-77)$ & $11 / 15$ & non-myxoid STTs & 0 & $\mathrm{HP}$ & $\begin{array}{l}\text { conventional } \\
\text { MRI } \\
\text { IVIM-DWI } \\
\text { DKI }\end{array}$ & $\begin{array}{l}\text { manually/solid } \\
\text { tumor area }\end{array}$ & $\begin{array}{l}\text { IVIM: ADCstd, } \\
\text { D, D*f } \\
\text { DKI:MK MD }\end{array}$ \\
\hline $\begin{array}{l}\text { Ogawa M(20) } \\
2017\end{array}$ & Japan & Pros & $43(25 / 18)$ & $62(18-90)$ & $27 / 16$ & non-myxoid STTs & 0 & $\begin{array}{l}\text { HP+follow } \\
\text { up }\end{array}$ & $\begin{array}{l}\text { conventional } \\
\text { MRI } \\
\text { DKI }\end{array}$ & $\begin{array}{l}\text { manually/solid } \\
\text { tumor area }\end{array}$ & $\begin{array}{l}\mathrm{MK} \\
\mathrm{ADC}_{\min }\end{array}$ \\
\hline
\end{tabular}

*Studies included all MRI technique, but we only extracted the DWI part when extract data.

MTTs: malignant soft tissue tumors; Retro: retrospective; Pros: prospective; All STTs: all soft tissue tumors; non-myxoid STTS: non-myxoid soft tissue tumors; ADC min: minimal ADC value; $\mathrm{ADC}_{\text {mean }}$ : mean $\mathrm{ADC}$ value; $\mathrm{ADCmax}$ : maximal ADC value; ADCratio: dividing tumor minimal ADC by muscle ADC values; $\mathrm{M} / \mathrm{F}$ : male/female; $\mathrm{M} / \mathrm{B}$ : malignant/benign.

\section{Imaging characteristic}

Table 2 provides an overview of the MRI characteristics reported in the 18 eligible studies. The MR examinations were performed on a $1.5 \mathrm{~T}$ scanner in $8(44.4 \%)$ studies and a $3.0 \mathrm{~T}$ scanner in $10(55.6 \%)$ studies, which evaluated the diagnostic performance of DWI with $b$ values ranging from 2 to 10 . ADC maps were generated from DWI in the maximal b-value ranging from $300 \mathrm{~s} / \mathrm{mm}^{2}$ to $2100 \mathrm{~s} / \mathrm{mm}^{2}$. Seven $(38.9 \%)$ studies used $\mathrm{ADC}_{\min }$ as the differentiation criterion with a cut-off value ranging from $0.8-1.9$, and $11(61.1 \%)$ studies used $\mathrm{ADC}_{\text {mean }}$ as the differentiation criterion with a cut-off value ranging from 1.09-1.6. 
Three (16.7\%) studies with IVIM DWI used all four IVIM parameters, including ADC, D, $\mathrm{D}^{*}$, and $\mathrm{f}$, and 1 $(5.56 \%)$ study only used two IVIM parameters, including ADC and D. Two (11.1\%) studies reporting DKI used MK. In addition, 3 (16.7\%) studies evaluated the diagnostic performance of ADC combined with conventional MRI.

\section{Quality of included studies}

Sixteen $(88.9 \%)$ of the 18 included studies with 5 or more than 5 terms were regarded as low risk, and they were finally regarded as high quality; as a result, the quality of all studies was high. The detailed results for the QUADAS-2 scores for every study can be found in Figure 2.
In the patient selection domain, 1 (5.6\%) study did not clearly describe the methods for patient selection, and $4(22.2 \%)$ studies did not clearly report inappropriate exclusion. In the index test domain, 6 (33.3\%) studies did not clearly report the threshold used, and $2(11.1 \%)$ studies did not clearly report the blinding of the reference standard from MRI results. For the reference test domain, $4(22.2 \%)$ studies were unclear, as no information was provided on whether the radiologists were blinded to the reference standard. For the flow and timing domain, 1 (5.6\%) study was unclear because the time interval between MRI and the reference standard was not reported.

Table 2. Imaging parameters of included studies

\begin{tabular}{|c|c|c|c|c|c|c|c|c|c|}
\hline Author, year & MRI vendor & $\begin{array}{l}\text { Strength } \\
\text { (T) }\end{array}$ & coil * & Sequence type & b-values (s/mm2) & TR/TE & Matrix size * & $\begin{array}{l}\text { Slice } \\
\text { thicknes } \\
\mathrm{s}(\mathrm{mm}) \text { * }\end{array}$ & $\mathrm{FOV}(\mathrm{mm} 2)$ * \\
\hline Lee SY (32) 2016 & Verio; Siemens & 3.0 & $\begin{array}{l}\text { phased-array } \\
\text { /eight-channel } \\
\text { extremity coil }\end{array}$ & SS-SE-EPI & 0.300 .800 .1400 & $5000-8700 / 71-85$ & $64 \times 45-20 \times 128$ & $2-5$ & $80-220$ \\
\hline $\begin{array}{l}\text { Del Grande F (36) } \\
2016\end{array}$ & $\begin{array}{l}\text { Verio, Skyra, Trio } \\
\text { Siemens }\end{array}$ & 3.0 & $\begin{array}{l}\text { phased-array } \\
\text { /eight-channel } \\
\text { extremity coil }\end{array}$ & SS-EPI & 50.400 .800 & $4500-7600 / 80-84$ & NA & NA & NA \\
\hline $\begin{array}{l}\text { Bonarelli C }{ }^{(33)} \\
2014\end{array}$ & Signa HDxt, GE & 1.5 & dedicated coils & SS-PGSE-EPI & 0.600 & $5000 /$ minimal & $128 \times 80$ & * & * \\
\hline $\begin{array}{l}\text { Dodin G }{ }^{(34)} \\
2020\end{array}$ & Signa HDxt, GE & 1.5 & NA & SS-PGSE-EPI & 0.600 & $4000-6225 / 66-88$ & $128 \times 80-256 \times 256$ & $5-6$ & $\begin{array}{l}190 \times 260-484 \times 28 \\
0\end{array}$ \\
\hline $\begin{array}{l}\text { Hassanien OA } \\
\text { (35) } 2018\end{array}$ & Signa EXCITE GE & 1.5 & $\begin{array}{l}\text { body } \\
\text { phased-array } \\
\text { coil }\end{array}$ & SS-EPI & 0.500 .800 & $8000-8500 / 50-60$ & $128 \times 64$ & $4-10$ & $25-30$ \\
\hline $\begin{array}{l}\text { Romeih } \mathrm{M}^{(9)} \\
2018\end{array}$ & $\begin{array}{l}\text { Achieva XR } \\
\text { Philips }\end{array}$ & 1.5 & surface coil & SS-SE-EPI & 0.400 .800 & NA & $128 \times 64$ & $4-10$ & NA \\
\hline $\begin{array}{l}\text { Teixeira PAG }{ }^{(37)} \\
2016\end{array}$ & Signa HDxt, GE & 1.5 & dedicated coils & SS-PGSE-EPI & 0.600 & $5000 /$ minimal & $128 \times 80$ & 6 & * \\
\hline Song Y (38) 2017 & $\begin{array}{l}\text { Achieva TX; } \\
\text { Philips }\end{array}$ & 3.0 & RF coils & SS-SE-EPI & 0.400 .800 & NA/61-69 & $128 \times 128-256 \times 256$ & 5 & $160-350$ \\
\hline Razek A ${ }^{(28)} 2010$ & $\begin{array}{l}\text { Symphony, } \\
\text { Siemens }\end{array}$ & 1.5 & NA & $\begin{array}{l}\text { multislice } \\
\text { SE-EPI }\end{array}$ & 0.500 .1000 & $10,000 / 108$ & $256 \times 128$ & 5 & $250-300$ \\
\hline Lee JH (29) 2020 & $\begin{array}{l}\text { Intera Achieva/ } \\
\text { Ingenia Philips }\end{array}$ & 3.0 & RF coils & SS-SE-EPI & 0.400 .800 & $5,000 / 61-69$ & $128 \times 128-256 \times 256$ & 5 & $160-350$ \\
\hline Robba T (30)2017 & $\begin{array}{l}\text { Signa Excite HD, } \\
\text { GE }\end{array}$ & 1.5 & NA & SS-EPI & 0.800 & NA & NA & NA & NA \\
\hline Choi YJ ${ }^{(31)} 2018$ & Tim Trio, Siemens & 3.0 & variable coils & SS-EPI & 0.50 .500 .800 .1400 & $4400-7100 / 56-88$ & $100-430$ & $2-7$ & $100-400$ \\
\hline Jeon JY ${ }^{(39)} 2016$ & $\begin{array}{l}\text { AchievaTM, } \\
\text { Philips }\end{array}$ & 3.0 & $\begin{array}{l}\text { dedicated } \\
\text { surface coils }\end{array}$ & SS-EPI & 0.400 .800 & $5000-5200 / 61-85$ & $70 \times 70-128 \times 128$ & $2.5-5$ & $100-250$ \\
\hline Wu H (15) $^{(15} 2017$ & $\begin{array}{l}\text { Achieva 1.5T, } \\
\text { Philips }\end{array}$ & 1.5 & $\begin{array}{l}\text { body or surface } \\
\text { coil }\end{array}$ & SS-PGSE-EPI & $\begin{array}{l}0.10 .20 .30 .40 .50 .75 .10 \\
0.150 .300 .500 .800\end{array}$ & $4500 / 65$ & $128 \times 136$ & 5 & $380 \times 380$ \\
\hline $\begin{array}{l}\text { Teixeira PAG } \\
2019\end{array}$ & $\begin{array}{l}\text { Discovery MR750, } \\
\text { GE }\end{array}$ & 3.0 & dedicated coils & SE-EPI & $\begin{array}{l}20.40 .60 .80 \\
100.200 .300 \\
500.700 .900\end{array}$ & $4000-17000 /<80$ & $96 \times 128$ & 5 & $\begin{array}{l}180 \times 180-500 \times 50 \\
0\end{array}$ \\
\hline Lee SK (40) 2020 & $\begin{array}{l}\text { MAGNETOM } \\
\text { Verio, Siemens }\end{array}$ & 3.0 & NA & SS-SE-EPI & $\begin{array}{l}0.25 .50 .75 .100 \\
200.300 .500 .800\end{array}$ & NA & NA & NA & NA \\
\hline Zhang X ${ }^{(41)} 2018$ & Skyra, Siemens & 3.0 & $\begin{array}{l}\text { eighteen-chann } \\
\text { el extremity coil }\end{array}$ & GRAPPA & $\begin{array}{l}\text { IVIM:0.10.20.30.40.50 } \\
.75 .100 .150 .200 .400 .8 \\
00, \\
1000,1500 \\
\text { DKI:0.100.700.1400.2 } \\
100\end{array}$ & $\begin{array}{l}\text { IVIM:3000/61 } \\
\text { DKI:3370/68 }\end{array}$ & $120 \times 120$ & 4 & $\begin{array}{l}\text { IVIM:203 ×203 } \\
\text { DKI:200 ×200 }\end{array}$ \\
\hline $\begin{array}{l}\text { Ogawa M } \\
\text { (20) } 2017\end{array}$ & Hitachi & 3.0 & NA & SE-EPI & 0.1000 .1500 .2000 & $3000 / 84$ & $64 \times 64$ & 5 & $250 \times 250$ \\
\hline
\end{tabular}

SS-EPI: single shot echo planar; SS-PGSE-EPI: single-shot pulsed gradient spin-echo echo planar; SS-SE-SEP: single shot, spin-echo echo planar; SE-EPI: spin-echo echo planar; multislice SE-EPI: multislice spin-echo echo planar; RF coils: radiofrequency coils.

*Depending on mass size and location, adapted to the patient anatomy and tumor size. 


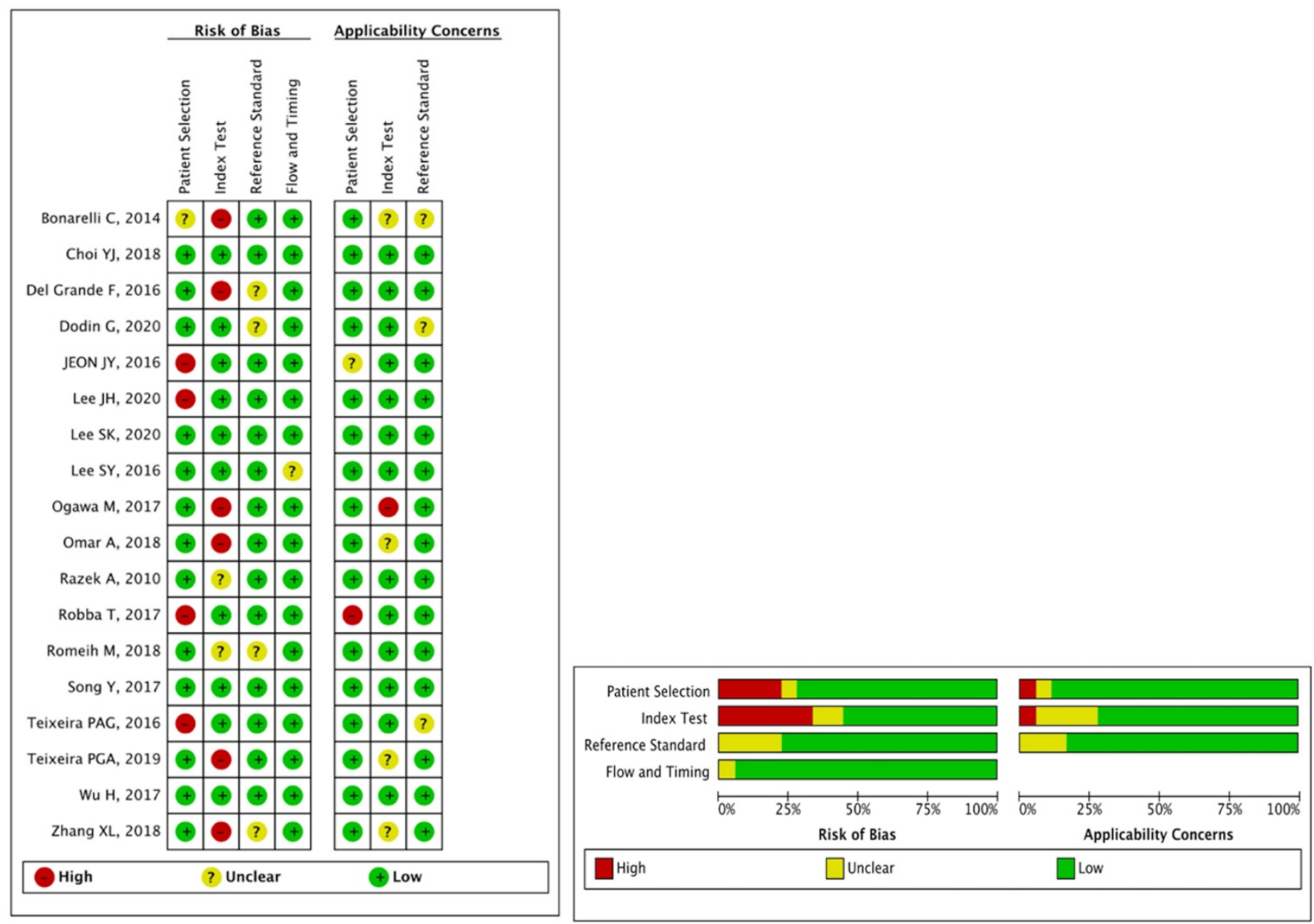

Figure 2. Summary of the risk of bias and applicability concerns across the included studies as assessed with QUADAS-2 forms.

\section{Diagnostic Performance of Quantitative Assessment of standard DWI}

The diagnostic results of standard DWI for benign and malignant STTs are shown in Table 3. A large range of mean $\mathrm{ADC}$ values for malignant $\left(0.74-1.58 \times 10^{-3} \mathrm{~mm}^{2} / \mathrm{s}\right)$, and benign lesions $\left(0.97-1.92 \times 10^{-3} \mathrm{~mm}^{2} / \mathrm{s}\right)$ was reported. The SEN and SPE of the included diagnostic results ranged from 0.53 to 1.0 and from 0.30 to 0.95 , respectively. The threshold effect was absent since the corresponding correlation coefficient was 0.047 between the logit of SEN and the logit of (1-SPE). The pooled SEN and SPE were 0.80 (95\% CI: $0.77-0.82)$ and 0.63 (95\% CI: $0.60-0.67)$, respectively. The forest plots are shown in Figure 3. The $Q$ test indicated the presence of heterogeneity $(\mathrm{Q}=45.07 ; p=0.01)$, and the $I^{2}$ value of $42.3 \%$ revealed moderate heterogeneity. However, SEN $\left(I^{2}=63.9 \%\right)$ and SPE $\left(I^{2}=80.2 \%\right)$ indicated significant heterogeneity. The DOR was 8.61 (95\% CI 6.93-10.72). The AUC was 0.806, and the SROC curve is shown in Figure 4. The results revealed the good diagnostic performance of ADC derived from DWI in differentiating benign and malignant STTs. When combined with conventional MRI in 3 studies [32, 33, 39], the AUCs increased to $0.97,0.66$, and 0.91 , and the AUC of the SROC curve was 0.99, indicating perfect diagnostic performance.

\section{Meta-regression and subgroup analyses}

We next carried out meta-regression and subgroup analyses to explore the possible sources of heterogeneity, and the results of meta-regression and subgroup analyses are summarized in Table 4 . Among the covariates that were considered a potential source of heterogeneity for sensitivity, the percentage of myxoid malignant tumors $(P=0.041)$, magnet strength $(P=0.019)$, study design $(P=0.00)$, and ROI placement $(P=0.018)$ were significant factors. Specifically, retrospective studies, studies that included myxoid malignant tumors less than $10 \%$, studies that used a magnet strength of $3.0 \mathrm{~T}$, and studies in which the ROI was not manually placed over the solid portion of the tumor reported higher SEN, SPE, DOR, and AUC values than the other subgroups. 

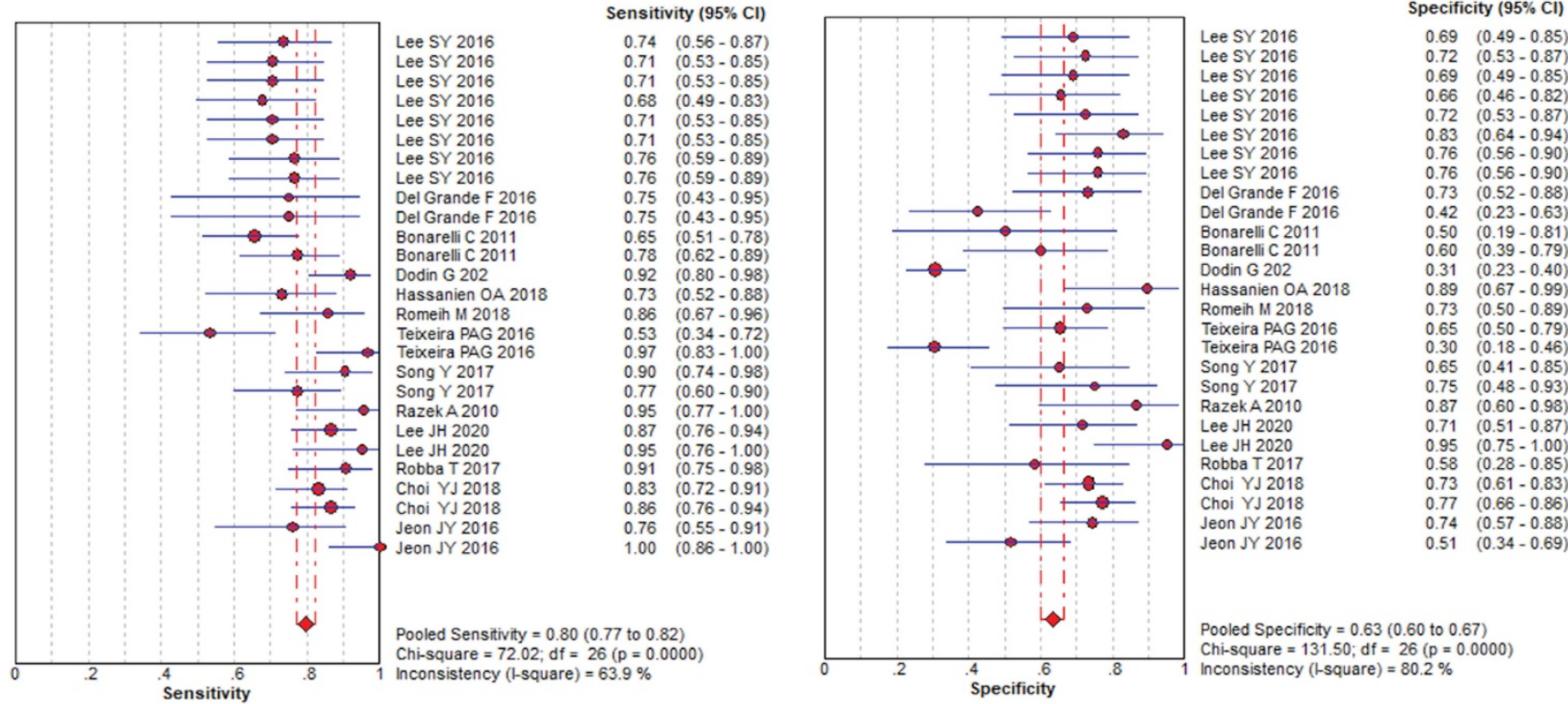

Figure 3. Pooled estimates of diagnostic performance of ADC values derived from DWI to differentiate benign and malignant STTs.

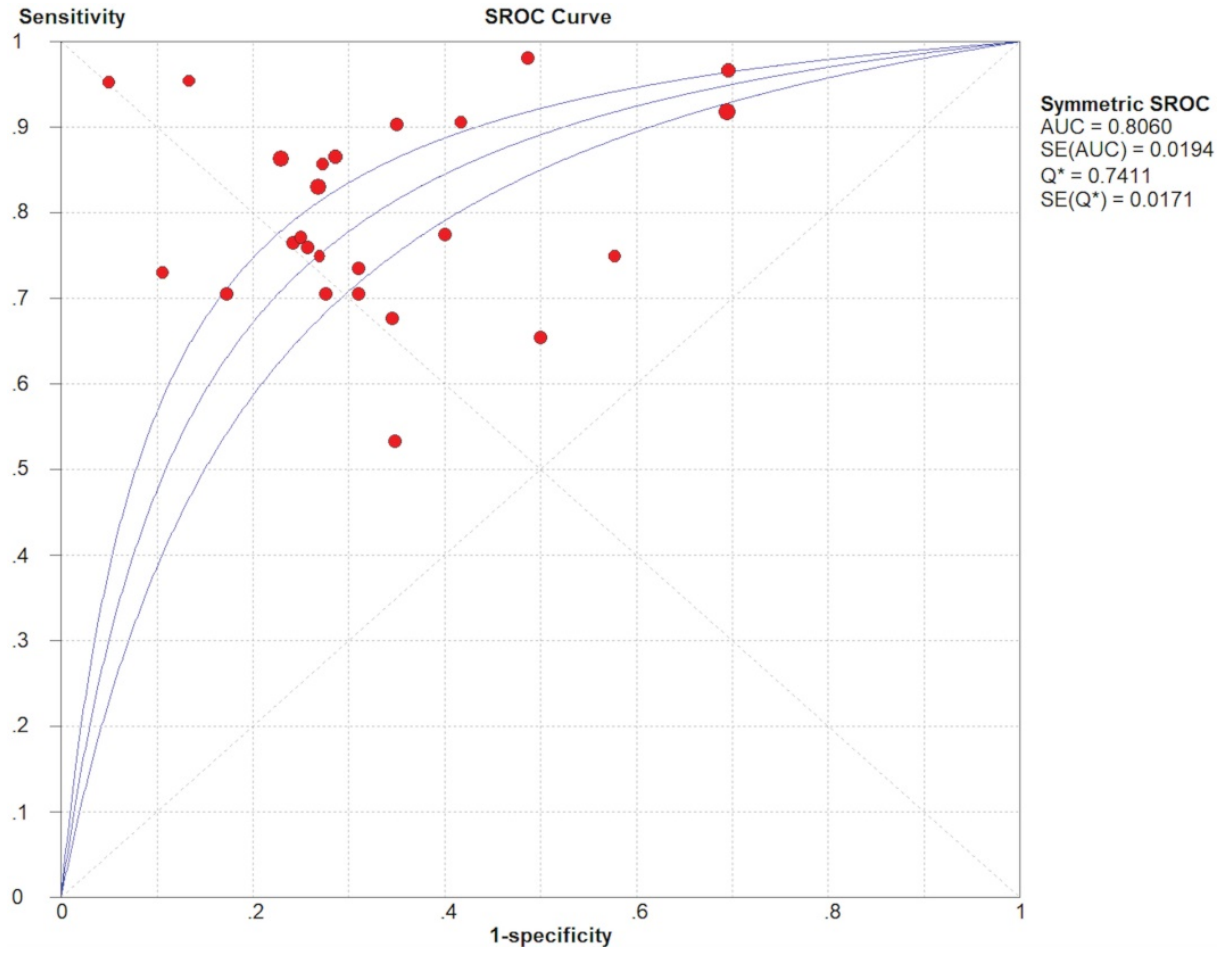

Figure 4. Summary receiver operating characteristic (SROC) curve of diagnostic performance of ADC values derived from DWI to differentiate benign and malignant STTs.

Table 3. Diagnostic results of ADC for benign and malignant soft tissue tumors

\begin{tabular}{|c|c|c|c|c|c|c|c|c|c|}
\hline Author, year & b-values (s/mm²) & $\begin{array}{l}\mathrm{ADC} \\
\text { measurements }\end{array}$ & $\begin{array}{l}\text { Cutoff values } \\
\left(\times 10^{-3} \mathrm{~mm}^{2} / \mathrm{s}\right)\end{array}$ & $\mathrm{TP}$ & FP & FN & $\mathrm{TN}$ & $\begin{array}{l}\text { ADC of benign tumors } \\
\left(\times 10^{-3} \mathrm{~mm}^{2} / \mathrm{s}\right)\end{array}$ & $\begin{array}{l}\text { ADC of Malignant } \\
\text { tumors }\left(\times 10^{-3} \mathrm{~mm}^{2} / \mathrm{s}\right)\end{array}$ \\
\hline \multirow[t]{8}{*}{ Lee SY, 2016} & 0.300 & $\mathrm{ADC}_{\text {mean }}$ & 1.60 & 25 & 9 & 9 & 20 & $1.92 \pm 0.63$ & $1.30 \pm 0.55$ \\
\hline & 0.800 & $\mathrm{ADC}_{\text {mean }}$ & 1.30 & 24 & 8 & 10 & 21 & $1.60 \pm 0.53$ & $1.09 \pm 0.49$ \\
\hline & 0.1400 & $\mathrm{ADC}_{\text {mean }}$ & 1.10 & 24 & 9 & 10 & 20 & $1.35 \pm 0.46$ & $0.94 \pm 0.44$ \\
\hline & 0.300 .800 .1400 & $\mathrm{ADC}_{\text {mean }}$ & 1.10 & 23 & 10 & 11 & 19 & $1.31 \pm 0.44$ & $0.94 \pm 0.44$ \\
\hline & 0.300 & $\mathrm{ADC}_{\min }$ & 1.30 & 24 & 8 & 10 & 21 & $1.70 \pm 0.62$ & $1.04 \pm 0.49$ \\
\hline & 0.800 & $\mathrm{ADC}_{\min }$ & 1.00 & 24 & 5 & 10 & 24 & $1.43 \pm 5.24$ & $0.86 \pm 0.41$ \\
\hline & 0.1400 & $\mathrm{ADC}_{\min }$ & 0.90 & 26 & 7 & 8 & 22 & $1.22 \pm 0.45$ & $0.76 \pm 0.37$ \\
\hline & 0.300 .800 .1400 & $\mathrm{ADC}_{\min }$ & 0.90 & 26 & 7 & 8 & 22 & $1.19 \pm 4.23$ & $0.76 \pm 0.39$ \\
\hline Del Grande F 2016 & $50,400,800$ & $\mathrm{ADC}_{\min }$ & 0.80 & 9 & 7 & 3 & 19 & NA & NA \\
\hline
\end{tabular}




\begin{tabular}{|c|c|c|c|c|c|c|c|c|c|}
\hline & $50,400,800$ & $\mathrm{ADC}_{\text {mean }}$ & 1.60 & 9 & 15 & 3 & 11 & NA & NA \\
\hline \multirow[t]{2}{*}{ Bonarelli C, 2014} & 0.600 & $\mathrm{ADC}_{\text {mean }}$ & 1.65 & 36 & 5 & 19 & 5 & $1.74 \pm 0.48$ & $1.58 \pm 0.47$ \\
\hline & 0.600 & $\mathrm{ADC}_{\min }$ & 1.28 & 31 & 10 & 9 & 15 & $1.51 \pm 0.59$ & $1.10 \pm 0.26$ \\
\hline Dodin G, 2020 & 0.600 & $\mathrm{ADC}_{\min }$ & 1.90 & 45 & 84 & 4 & 37 & $1.63 \pm 0.61$ & $1.30 \pm 0.44$ \\
\hline Hassanien OA, 2018 & 0.500 .800 & $\mathrm{ADC}_{\text {mean }}$ & 1.24 & 19 & 2 & 7 & 17 & NA & NA \\
\hline Romeih M, 2018 & 0.400 .800 & $\mathrm{ADC}_{\text {mean }}$ & 1.10 & 24 & 6 & 4 & 16 & $1.43 \pm 0.56$ & $0.74 \pm 0.18$ \\
\hline Teixeira PAG & $\mathrm{a} 0.600$ & $\mathrm{ADC}_{\min }$ & 1.19 & 16 & 16 & 14 & 30 & NA & NA \\
\hline 2016 & $\mathrm{~b} 0.600$ & $\mathrm{ADC}_{\min }$ & 1.68 & 29 & 32 & 1 & 14 & NA & NA \\
\hline \multirow[t]{2}{*}{ Song Y, 2017} & 0.400 .800 & $\mathrm{ADC}_{\text {mean }}$ & 1.13 & 28 & 7 & 3 & 13 & $1.60 \pm 0.48$ & $1.24 \pm 0.46$ \\
\hline & 0.400 .800 & $\mathrm{ADC}_{\min }$ & 0.63 & 27 & 4 & 8 & 12 & $0.97 \pm 0.41$ & $0.94 \pm 0.35$ \\
\hline Razek A, 2010 & 0.500 .1000 & $\mathrm{ADC}_{\text {mean }}$ & 1.34 & 21 & 2 & 1 & 13 & $1.54 \pm 0.03$ & $1.02 \pm 0.30$ \\
\hline \multirow[t]{2}{*}{ Lee JH, 2020} & c0.400.800 & $\mathrm{ADC}_{\text {mean }}$ & 1.36 & 58 & 8 & 9 & 20 & $1.62 \pm 0.50$ & $1.13 \pm 0.47$ \\
\hline & $\mathrm{d} 0.400 .800$ & $\mathrm{ADC}_{\text {mean }}$ & 0.91 & 20 & 1 & 1 & 19 & $1.38 \pm 0.40$ & $0.94 \pm 0.23$ \\
\hline Robba T, 2017 & 0.800 & $\mathrm{ADC}_{\text {mean }}$ & 1.45 & 29 & 5 & 3 & 7 & NA & NA \\
\hline \multirow[t]{2}{*}{ Choi YJ, 2018} & $0,50,500,800,1400$ & $\mathrm{ADC}_{\min }$ & 0.94 & 54 & 19 & 11 & 52 & $1.44 \pm 0.46$ & $0.90 \pm 0.40$ \\
\hline & $0,50,500,800,1400$ & $\mathrm{ADC}_{\text {mean }}$ & 1.18 & 57 & 16 & 9 & 54 & $1.13 \pm 0.42$ & $0.77 \pm 0.36$ \\
\hline \multirow[t]{2}{*}{ Jeon JY,2016 } & $\mathrm{e} 0.400 .800$ & $\mathrm{ADC}_{\text {mean }}$ & 1.09 & 19 & 9 & 6 & 26 & $1.39 \pm 0.51$ & $0.87 \pm 0.36$ \\
\hline & f 0.400 .800 & $\mathrm{ADC}_{\text {mean }}$ & 1.49 & 25 & 17 & 0 & 18 & $1.56 \pm 0.61$ & $1.01 \pm 0.33$ \\
\hline
\end{tabular}

ab: Result from different cut off value; cd: result from all STTs and non-myxoid STTs respectively; ef: result from different ROI placement.

Table 4. Results of Meta-regression and Subgroup Analyses for benign and malignant soft tissue tumors

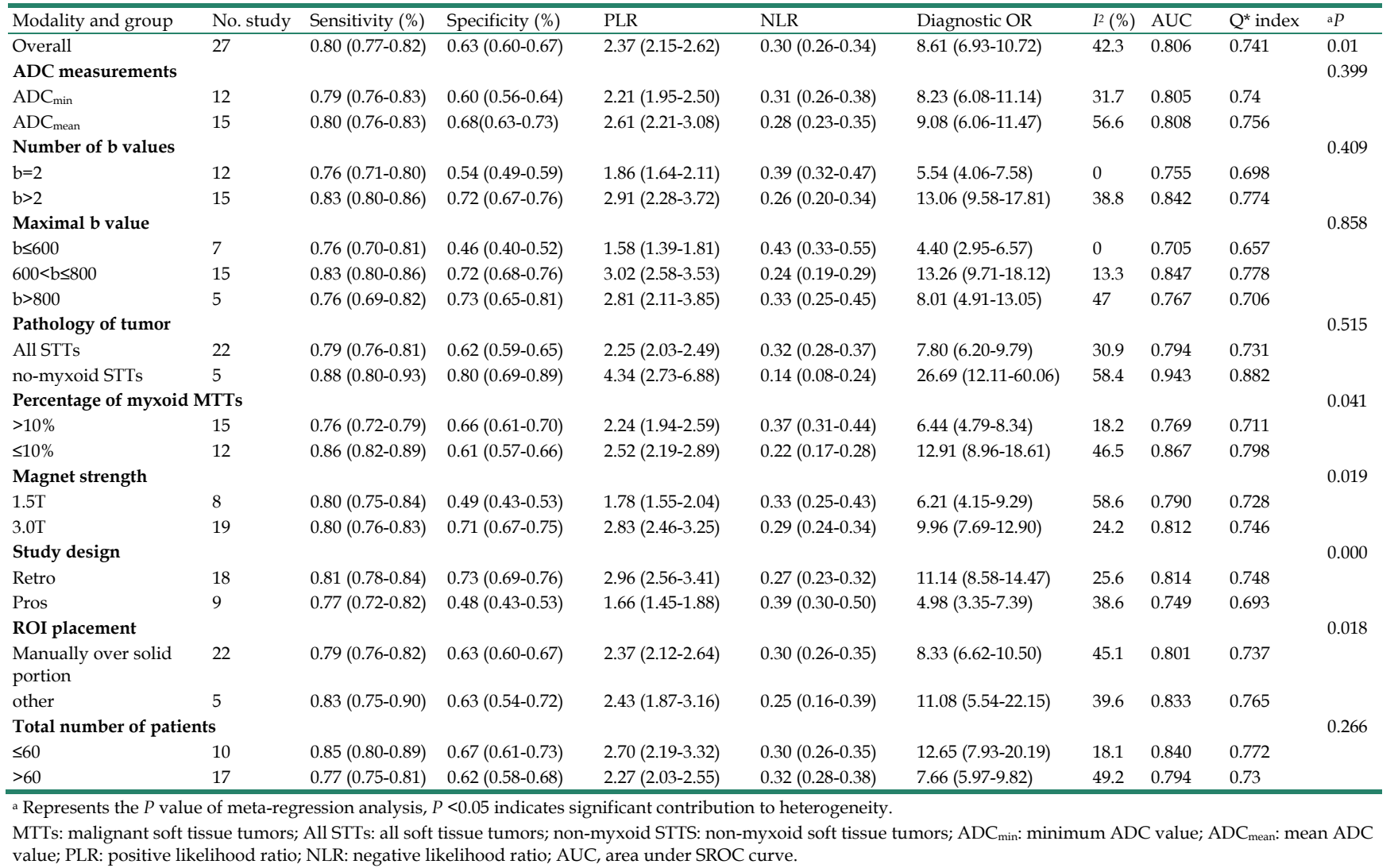

The other factors, including the ADC measurements, number of $b$ values, maximal $b$ value, total number of patients, and pathology of the tumor, were insignificantly contributors to the heterogeneity. However, studies that only included non-myxoid tumors, studies that chose maximal $b$ values ranging from 600 to $800 \mathrm{~s} / \mathrm{mm}^{2}$, and studies that included more than $2 \mathrm{~b}$ values also reported slightly higher SEN, SPE, DOR, and AUC values.

\section{Diagnostic Performance of Quantitative Assessment of IVIM-DWI}

Table 5 shows the diagnostic results of IVIM DWI for benign and malignant STTs. A large range of mean ADC, D, D*, and $\mathrm{f}$ values was reported. The threshold effect was observed for parameters ADC and $\mathrm{D}$, since the correlation coefficients between the logit of SEN and the logit of (1-SPE) were 0.771 and 0.771 , respectively, and the areas under the SROC curves (AUCs) were 0.859 and 0.874, respectively 
(shown in Figure 5). The $\mathrm{Q}$ test indicated the absence of heterogeneity $(\mathrm{Q}=2.07, P=0.839 ; \mathrm{Q}=1.06, P=0.957$; respectively) and the SEN $\left(I^{2}=47.5 \%\right.$ and $41.8 \%$, respectively) and SPE ( $I^{2}=39.2 \%, 0 \%$, respectively) exhibited moderate heterogeneity. The results revealed the good diagnostic performance of ADC and $\mathrm{D}$ derived from IVIM in differentiating benign and malignant STTs.

However, the threshold effect was absent for the parameters $D^{*}$ and $f$, since the correlation coefficients were -0.30 and 0.40 , respectively. The $Q$ test exhibited no significant heterogeneity $(\mathrm{Q}=7.29, P=0.12 ; \mathrm{Q}=3.58$, $P=0.46$; respectively). However, the pooled SEN was 0.68 (95\% CI: $0.58-0.76)$ and 0.52 (95\% CI: 0.43-0.61), the SPE was 0.68 (95\% CI: 0.59-0.75) and 0.67 (95\% CI: 0.59-0.74), and the AUCs were 0.736 and 0.573, respectively. The results were lower than other parameters and may indicate a failed-to-fair diagnostic performance in differentiating benign and malignant STTs.

Table 5. Diagnostic results of IVIM for benign and malignant soft tissue tumors

\begin{tabular}{|c|c|c|c|c|c|c|c|c|c|}
\hline Author year & b-values (s/mm²) & IVIM parameters & $\begin{array}{l}\text { Cutoff values } \\
\left(\times 10^{-3} \mathrm{~mm}^{2} / \mathrm{s}\right)\end{array}$ & $\mathrm{TP}$ & FP & $\mathrm{FN}$ & $\mathrm{TN}$ & $\begin{array}{l}\text { Benign tumors } \\
\left(\times 10^{-3} \mathrm{~mm}^{2} / \mathrm{s}\right)\end{array}$ & $\begin{array}{l}\text { Malignant tumors } \\
\left(\times 10^{-3} \mathrm{~mm}^{2} / \mathrm{s}\right)\end{array}$ \\
\hline \multirow[t]{4}{*}{$\mathrm{Wu} \mathrm{H}, 2017$} & \multirow{4}{*}{$\begin{array}{l}0,10,20,30,50,100,200,300, \\
500,800\end{array}$} & $\mathrm{ADC}$ & 10.24 & 10 & 5 & 4 & 39 & $1.62 \pm 0.06$ & $1.28 \pm 0.08$ \\
\hline & & $\mathrm{D}$ & 10.13 & 11 & 10 & 3 & 34 & $1.35 \pm 0.05$ & $1.06 \pm 0.06$ \\
\hline & & $D^{*}$ & 1190 & 12 & 11 & 2 & 23 & $124.96 \pm 3.96$ & $144.40 \pm 6.65$ \\
\hline & & $\mathrm{f}$ & 90.85 & 4 & 3 & 10 & 41 & $14.70 \pm 0.81$ & $14.44 \pm 1.42$ \\
\hline \multirow{8}{*}{$\begin{array}{l}\text { Teixeira, PAG } \\
2019\end{array}$} & \multirow{4}{*}{$\begin{array}{l}\text { a20, } 40,60,80,100,200,300, \\
500,700,900\end{array}$} & $\mathrm{ADC}$ & 125 & 18 & 5 & 11 & 32 & $1.63 \pm 0.05$ & $1.23 \pm 0.05$ \\
\hline & & $\mathrm{D}$ & 96 & 15 & 3 & 14 & 32 & $1.54 \pm 0.05$ & $1.12 \pm 0.05$ \\
\hline & & $\mathrm{D}^{*}$ & 992 & 18 & 7 & 11 & 28 & $84.2 \pm 76.2$ & $132.1 \pm 103.1$ \\
\hline & & f & 85 & 16 & 18 & 13 & 17 & $0.1 \pm 0.1$ & $0.1 \pm 0.1$ \\
\hline & \multirow{4}{*}{$\begin{array}{l}\text { b20, } 40,60,80,100,200,300, \\
500,700,900\end{array}$} & $\mathrm{ADC}$ & 125 & 18 & 5 & 4 & 25 & $1.51 \pm 0.04$ & $1.94 \pm 0.04$ \\
\hline & & $\mathrm{D}$ & 99 & 16 & 3 & 6 & 27 & $1.42 \pm 0.04$ & $0.94 \pm 0.05$ \\
\hline & & $\mathrm{D}^{*}$ & 750 & 18 & 9 & 4 & 21 & $90.6 \pm 78.6$ & $153.2 \pm 108.4$ \\
\hline & & f & 90 & 11 & 12 & 11 & 18 & $0.1 \pm 0.06$ & $0.1 \pm 0.04$ \\
\hline \multirow[t]{8}{*}{ Lee SK, 2020} & \multirow{4}{*}{$\begin{array}{l}a 0,25,50,75,100,200,300, \\
500,800\end{array}$} & $\mathrm{ADC}$ & 1.31 & 23 & 8 & 8 & 22 & $1.47 \pm 0.35$ & $1.17 \pm 0.49$ \\
\hline & & $\mathrm{D}$ & 1.19 & 22 & 5 & 9 & 25 & $1.41 \pm 0.37$ & $1.13 \pm 0.50$ \\
\hline & & $\mathrm{D}^{*}$ & 274 & 18 & 13 & 13 & 17 & $297 \pm 96$ & $258 \pm 83$ \\
\hline & & f & 82 & 17 & 13 & 14 & 17 & $1.01 \pm 0.58$ & $0.83 \pm 0.36$ \\
\hline & \multirow{4}{*}{$\begin{array}{l}\text { b0, } 25,50,75,100,200,300 \text {, } \\
500,800\end{array}$} & $\mathrm{ADC}$ & 1.26 & 20 & 5 & 3 & 14 & $1.37 \pm 0.24$ & $0.98 \pm 0.30$ \\
\hline & & $\mathrm{D}$ & 1.18 & 20 & 4 & 3 & 15 & $1.29 \pm 0.25$ & $0.94 \pm 0.29$ \\
\hline & & $\mathrm{D}^{*}$ & 279 & 15 & 8 & 8 & 11 & $312 \pm 114$ & $266 \pm 92$ \\
\hline & & f & 88 & 14 & 6 & 9 & 13 & $114 \pm 61$ & $88 \pm 36$ \\
\hline \multirow[t]{2}{*}{ Zhang XL 2018} & $0,10,20,30,40,50,75,100,150,2$ & $\mathrm{ADC}$ & 1.33 & 8 & 7 & 0 & 11 & $1.90 \pm 0.43$ & $1.27 \pm 0.38$ \\
\hline & $00,400,800,1000,1500$ & $\mathrm{D}$ & 1.42 & 14 & 1 & 5 & 6 & $1.71 \pm 0.45$ & $1.04 \pm 0.35$ \\
\hline
\end{tabular}

a.b Results from all STTs and non-myxoid STTs respectively.
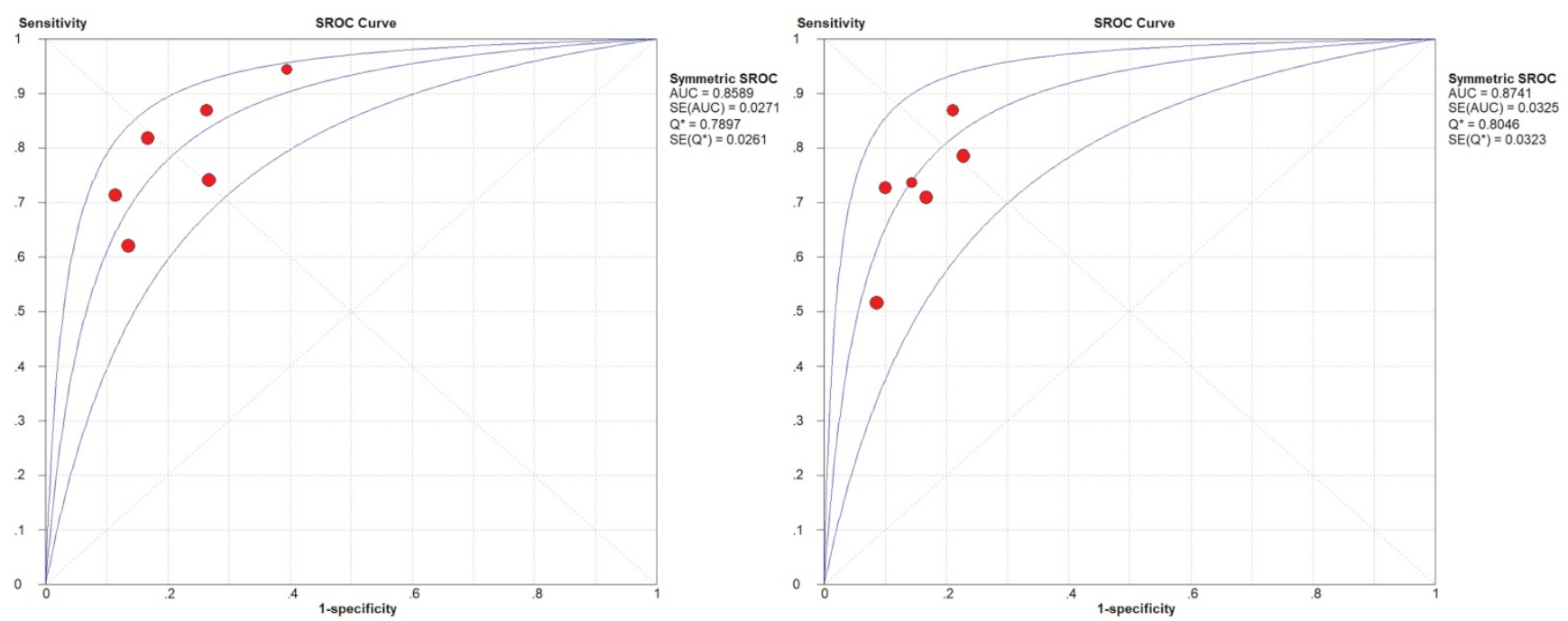

Figure 5. Summary receiver operating characteristic (SROC) curve of diagnostic performance of ADC and D values derived from IVIM-DWI to differentiate benign and malignant STTs. 


\section{Diagnostic Performance of Quantitative Assessment of DKI}

Two studies with DKI used the parameter MK. One study chose 4 values for the diffusion factor $b(0$, 1000, 1500, and $2000 \mathrm{~s} / \mathrm{mm}^{2}$ ) in combination for diagnosis. The mean $( \pm S D)$ MK values were $0.49 \pm 0.17$ and $1.14 \pm 0.30$ for benign and malignant tumors, respectively. At a cut-off of $\mathrm{MK}=0.81$, the SPE and SEN for the diagnosis of malignant tumors were 96.3 and $93.8 \%$, respectively. The AUC was 0.97 for MK. Another study chose 5 values for the diffusion factor $b$ $\left(0,100,700,1400\right.$ and $\left.2000 \mathrm{~s} / \mathrm{mm}^{2}\right)$ in combination for diagnosis. The mean $( \pm S D)$ MK values were $0.45 \pm 0.97$ and $0.82 \pm 0.56$ for benign and malignant tumors, respectively. At a cut-off of $\mathrm{MK}=0.60$, the SPE and SEN for the diagnosis of malignant tumors were 60 and $100 \%$, respectively. The AUC was 0.89 for MK.

\section{Discussion}

To our knowledge, our meta-analysis is the first to systematically compare all relevant standard DWI, IVIM DWI and advanced non-Gaussian diffusion techniques for DKI to evaluate the diagnostic performance in distinguishing benign and malignant musculoskeletal STTs. According to our search, few reviews pertain to musculoskeletal STTs. The aims of these reviews are divided into two types: one was to analyze DWI findings of different histological types of tumors and to compare the ADC values acquired, and the other was to review the various techniques of DWI acquisition and the utility of qualitative and quantitative methods of image interpretation, with an emphasis on optimal methods for ADC measurement $[7,42-45]$. There was only one meta-analysis evaluated the diagnostic potential of ADC values obtained from standard DWI in distinguishing malignant and benign STTs, which was estimated by the standard mean difference (SMD) [46].

The results of our meta-analysis revealed that standard DWI displays good diagnostic performance for discriminating between benign and malignant musculoskeletal STTs. However, a number of heterogeneities between studies of ADC need further consideration. By performing a meta-regression analysis, DWI performed at higher field strengths using 3.0 T MR systems was more effective than DWI performed using a $1.5 \mathrm{~T}$ system because the signal-to-noise ratio is influenced by the strength of the magnetic field, with higher fields producing a better signal-to-noise ratio and therefore a better quality of MRI for accurately drawing an ROI of the tumor $[27,46]$. The difference in the locations of ROIs also led to heterogeneity. Jeon, JY et al [39] reported that the AUCs of both ADC values extracted from the solid portion and whole tumor were nearly identical, but the ADC values extracted from the solid portion exhibited better diagnostic accuracy but lower sensitivity. The results of the subgroup analysis conducted in our study did not contradict this conclusion. Our study reported that setting the ROIs manually in the solid portion to extract ADC values had a lower AUC and sensitivity, which may make the ADCs unavailable to systematically clarify the characteristics and heterogeneity[26], but this result was not reliable, because of the unstandardized technique, further studies will be needed to clarify the accuracy of this conclusion. The percentage of myxoid malignant STTs in every study might also be an important source of heterogeneity. Myxoid components of STTs are a potential source of inconsistency in the discrimination of malignant STTs from benign STTs, and significantly higher ADC values have been observed in myxoid-containing lesions $[10,30,38]$, which may decrease the accuracy of ADC. Therefore, in our results, compared with the subgroup with a percentage of myxoid malignant tumors greater than $10 \%$, the less than $10 \%$ subgroup showed a better diagnostic performance with a higher AUC and SEN values.

By other subgroup analysis, we found that maximal $b$ value $(600<b \leq 800)$, the number of $b$ value $(>2)$, pathology of tumor (non-myxoid tumor) and the ADC measurement were insignificantly contributed to the heterogeneity, but also have clinical diagnosis value, and probably affect test performance. The use of a higher $b$ value for DWI scans has been suggested to result in ADC values with a lower effect of blood perfusion and a better response to the diffusion of water molecules within the tissue [27], and the use of more $b$ values might achieve better separation. Although the use of at least three values ranging from $150-900 \mathrm{~mm}^{2} / \mathrm{s}$ was advocated in the Consensus Conference held in Toronto in 2008 [47], the optimal number and scale of $b$ value are still controversial [31, 32], and our results may provide reference values for clinical applications to some extent. In the subgroup analysis, the best results were obtained from the study group in which the tumor pathology was non-myxoid tumors, with an AUC of 0.94. As mentioned above, ADC values for the percentage of myxoid malignant tumors may be affected by the myxoid matrix within the tumor, which makes a radiological diagnosis based on ADC values alone quite difficult $[6,29]$. However, most myxoid tumors have suggestive characteristics on conventional MRI, appearing as "fluid-like" lesions with very high signal intensity on T2-weighted images, low signal intensity on T1-weighted images and variable enhancement after gadolinium injection [37, 48]. Thus, the combination of these techniques in conventional MRI may lead to 
fewer misdiagnoses. As shown in our meta-analysis, 3 studies [32, 33, 39] analysed the diagnostic performance of DWI combined with conventional MRI, and the AUC of the SROC curve was 0.99, which was significantly higher than DWI alone and indicated perfect diagnostic performance. In clinical practice, for tumors with no typical myxoid characteristics according to conventional MRI, the ADC technique can be added to increase the diagnostic SEN. Moreover, no consensus has been reached regarding the most useful ADC measurements. A previous review [42] reported that the $\mathrm{ADC}_{\min }$ value had a better diagnostic performance for discriminating STTs because it may provide more insights into the cellular composition, while the $\mathrm{ADC}_{\text {mean }}$ value may be diluted because of myxoid, cystic or fewer cellular regions included in the ROI. In contrast, our study revealed that the pooled SEN, SPE and $\mathrm{AUC}$ of both $\mathrm{ADC}_{\text {min }}$ and $\mathrm{ADC}_{\text {mean }}$ values were nearly identical, which may be important for radiologists when the ADC of DWI is applied in daily clinical practice.

Furthermore, our meta-analysis revealed that IVIM DWI-derived D and ADC values showed good diagnostic performance for differentiating malignant from benign STTs, and D would be slightly better than ADC because $\mathrm{D}$ eliminates the contributions of tissue perfusion to reflect tissue diffusivity more precisely than ADC[49]. Our results contradicted a previous study by $\mathrm{Wu} \mathrm{H}$ and Lee SK et al $[15,40]$ showing that the AUCs of ADC were slightly better than those of D based on the ROC analysis. We also noted that the $\mathrm{ADC}$ and $\mathrm{D}$ values gradually decreased from benign to malignant tumors, in which water molecule diffusion was more impeded due to the increase in cell density and decrease in the extracellular space [15, 17, 50]. However, IVIM-derived $\mathrm{D}^{*}$ and $\mathrm{f}$ failed to show fair diagnostic performance. $\mathrm{D}^{*}$ is considered proportional to the mean capillary segment length and average blood velocity and may chiefly reflect tumor vascularity [15, 51] Although our results showed a distinct increase in $\mathrm{D}^{*}$ from benign to malignant soft tissue tumors, the diagnostic performance was fair. The $f$ value may correlate with the amount of normal angiogenesis with intact vessels in terms of basement membrane thickness and pericyte coverage and may be an indicator of intact vascular permeability. Perhaps due to variability in histological grades or varying tumor vascularity among the tumor types $[15,52]$, the diagnostic performance of the $f$ value was poor. Further studies with larger numbers of subjects are needed to confirm our findings.

According to previous studies, DKI parameters are sensitive to noise effects and the degradation of image quality that strongly influence nonbrain imaging, and the reliability may be insufficient, especially for nonbrain imaging [20,53]. Few DKI studies on musculoskeletal STTs have been conducted, and we only included 2 studies. They both revealed that the DKI-derived MK parameter showed good diagnostic performance for differentiating malignant from benign STTs, which was presumed to be correlated with the complexity of the tumor microenvironment [39]. However, the result is inconclusive due to the small number of publications, and these studies did not include patients with myxoid sarcoma or chondrosarcoma.

\section{Limitations}

Our study has some limitations that cannot be ignored. First, the overrepresentation of a sample may be a limitation of our pooled estimates because we included multiple sensitivities and specificities from the same authors investigating the same population. Second, our study did not include DTI studies. DTI is one of the conventional DWI techniques that evaluate the three-dimensional (3D) motion of protons in tissues, providing quantitative data on the amount and directionality of random movement of water molecules. DTI is a feasible technique that is mainly used to evaluate peripheral nerve tumors and soft tissue tumors arising around nerve structures [54, 55]. Therefore, these studies did not conform to our study selection criteria. Last, although the possible sources of heterogeneity were explored, the different MRI vendors, sequences, imaging parameters, anatomical locations and tumor physiologies and their potential impacts on conclusions should not be neglected; thus, well-conducted investigations using a standardized methodology are needed to confirm the utility of DWI for discriminating between benign and malignant STTs.

\section{Conclusions}

In conclusion, the DWI-derived ADC value and the IVIM DWI-derived D value might be an accurate tool for discriminating STTs, especially for non-myxoid SSTs; the use of more than two b values, a maximal b value between 600 and $800 \mathrm{~s} / \mathrm{mm}^{2}$, and high field strength $(3.0 \mathrm{~T})$ may optimize the diagnostic performance. The use of DWI combined with conventional MRI might help to improve the diagnostic accuracy. However, more high-quality studies with larger samples are warranted to verify a standardized methodology in clinical practice.

\section{Abbreviations}

MRI: Magnetic resonance imaging; DWI: diffusion-weighted imaging; IVIM: intravoxel 
incoherent motion; DKI: diffusion kurtosis imaging; STTs: soft tissue tumors; ADC: apparent diffusion coefficient; MK: mean kurtosis; $\mathrm{D}^{*}$ : pseudo diffusion coefficient; f: perfusion fraction; SEN: sensitivity; SPE: specificity; SROC: summary receiver operating characteristic; AUC: area under the curve; CI: confidence interval; DOR: diagnostic odds ratio; FN: false-negative; FP: false-positive; TN: true-negative; TP: true-positive; NLR: negative likelihood ratio; PLR: positive likelihood ratio; QUADAS: Quality Assessment of Diagnostic Accuracy Studies.

\section{Competing Interests}

The authors have declared that no competing interest exists.

\section{References}

1. Miwa S, Otsuka T. Practical use of imaging technique for management of bone and soft tissue tumors. J Orthop Sci. 2017; 22: 391-400.

2. Rochwerger A, Mattei JC. Management of soft tissue tumors of the musculoskeletal system. Orthopaedics and Traumatology: Surgery and Research. 2018; 104: S9-S17.

3. Ahlawat S, Fayad LM. Revisiting the who classification system of soft tissue tumours: Emphasis on advanced magnetic resonance imaging sequences. part 1. Polish Journal of Radiology. 2020; 85: e396-e408.

4. Knapp EL, Kransdorf MJ, Letson GD. Diagnostic imaging update: soft tissue sarcomas. Cancer control : journal of the Moffitt Cancer Center. 2005; 12: 22-6.

5. Vilanova JC, Woertler K, Narvaez JA, Barcelo J, Martinez SJ, Villalon M, et al. Soft-tissue tumors update: MR imaging features according to the WHO classification. Eur Radiol. 2007; 17: 125-38.

6. Nagata S, Nishimura H, Uchida M, Sakoda J, Tonan T, Hiraoka K, et al. Diffusion-weighted imaging of soft tissue tumors: usefulness of the apparent diffusion coefficient for differential diagnosis. Radiat Med. 2008; 26: 287-95.

7. Khoo MMY, Tyler PA, Saifuddin A, Padhani AR. Diffusion-weighted imaging (DWI) in musculoskeletal MRI: A critical review. Skeletal Radiol. 2011; 40: 665-81.

8. Bley TA, Wieben O, Uhl M. Diffusion-weighted MR imaging in musculoskeletal radiology: applications in trauma, tumors, and inflammation. Magn Reson Imaging Clin N Am. 2009; 17: 263-75.

9. Romeih M, Raafat T, Khalaf M, Sallam K. The diagnostic value of diffusionweighted magnetic resonance imaging in characterization of musculoskeletal soft tissue tumors. Egyptian Journal of Radiology and Nuclear Medicine. 2018; 49: 400-7

10. Maeda $\mathrm{M}$, Matsumine A, Kato $\mathrm{H}$, Kusuzaki $\mathrm{K}$, Maier SE, Uchida A, et al. Soft-tissue tumors evaluated by line-scan diffusion-weighted imaging: influence of myxoid matrix on the apparent diffusion coefficient. Journal of magnetic resonance imaging : JMRI. 2007; 25: 1199-204

11. Einarsdottir H, Karlsson M, Wejde J, Bauer HCF. Diffusion-weighted MRI of soft tissue tumours. Eur Radiol. 2004; 14: 959-63.

12. Lim HK, Jee $\mathrm{W}-\mathrm{H}$, Jung J-Y, Paek MY, Kim I, Jung C-K, et al. Intravoxel incoherent motion diffusion-weighted MR imaging for differentiation of benign and malignant musculoskeletal tumours at $3 \mathrm{~T}$. The British journal of radiology. 2018; 91: 20170636

13. Du J, Li K, Zhang W, Wang $S$, Song $\mathrm{Q}$ Liu A, et al. Intravoxel Incoherent Motion MR Imaging: Comparison of Diffusion and Perfusion Characteristics for Differential Diagnosis of Soft Tissue Tumors. Medicine (Baltimore). 2015; 94: e1028.

14. Le Bihan D, Breton E, Lallemand D, Aubin ML, Vignaud J, Laval-Jeantet M. Separation of diffusion and perfusion in intravoxel incoherent motion MR imaging. Radiology. 1988; 168: 497-505.

15. Wu $\mathrm{H}$, Zhang $\mathrm{S}$, Liang $\mathrm{C}$, Liu $\mathrm{Y}$, Mei $\mathrm{Y}$, Liu $\mathrm{H}$, et al. Intravoxel incoherent motion MRI for the differentiation of benign, intermediate, and malignant solid soft-tissue tumors. J Magn Reson Imaging. 2017; 46: 1611-8.

16. Jiang H, Chen J, Gao R, Huang Z, Wu M, Song B. Liver fibrosis staging with diffusion-weighted imaging: a systematic review and meta-analysis. Abdom Radiol (NY). 2017; 42: 490-501.

17. Kang KM, Lee JM, Yoon JH, Kiefer B, Han JK, Choi BI. Intravoxel incoherent motion diffusion-weighted MR imaging for characterization of focal pancreatic lesions. Radiology. 2014; 270: 444-53.

18. Baxter GC, Graves MJ, Gilbert FJ, Patterson AJ. A Meta-analysis of the Diagnostic Performance of Diffusion MRI for Breast Lesion Characterization. Radiology. 2019; 291: 632-41.

19. Iima M, Le Bihan D. Clinical Intravoxel Incoherent Motion and Diffusion MR Imaging: Past, Present, and Future. Radiology. 2016; 278: 13-32.
20. Ogawa $\mathrm{M}$, Kan $\mathrm{H}$, Arai N, Murai $\mathrm{T}$, Manabe $\mathrm{Y}$, Sawada $\mathrm{Y}$, et al. Differentiation between malignant and benign musculoskeletal tumors using diffusion kurtosis imaging. Skeletal Radiol. 2019; 48: 285-92.

21. Wu G, Liu X, Xiong $Y$, Ran J, Li X. Intravoxel incoherent motion and diffusion kurtosis imaging for discriminating soft tissue sarcoma from vascular anomalies. Medicine (Baltimore). 2018; 97: e13641.

22. Jiang R, Jiang J, Zhao L, Zhang J, Zhang S, Yao Y, et al. Diffusion kurtosis imaging can efficiently assess the glioma grade and cellular proliferation. Oncotarget. 2015; 6: 42380-93

23. Gondim Teixeira PA, Simon L, Sirveaux F, Marie B, Louis M, Hossu G, et al. Intravoxel incoherent motion MRI for the initial characterization of non-fatty non-vascular soft tissue tumors. Diagn Interv Imaging. 2020; 101: 245-55.

24. Frank RA, Bossuyt PM, McInnes MDF. Systematic Reviews and Meta-Analyses of Diagnostic Test Accuracy: The PRISMA-DTA Statement. Radiology. 2018; 289: 313-4.

25. Whiting PF, Rutjes AW, Westwood ME, Mallett S, Deeks JJ, Reitsma JB, et al. QUADAS-2: a revised tool for the quality assessment of diagnostic accuracy studies. Ann Intern Med. 2011; 155: 529-36.

26. Lu X, Xu W, Wei Y, Li T, Gao L, Fu X, et al. Diagnostic performance of DWI for differentiating primary central nervous system lymphoma from glioblastoma: a systematic review and meta-analysis. Neurol Sci. 2019; 40: 947-56.

27. Zong RL, Geng L, Wang X, Xie D. Diagnostic Performance of Apparent Diffusion Coefficient for Prediction of Grading of Pancreatic Neuroendocrine Tumors: A Systematic Review and Meta-analysis. Pancreas. 2019; 48: 151-60.

28. Razek A, Nada N, Ghaniem M, Elkhamary S. Assessment of soft tissue tumours of the extremities with diffusion echoplanar MR imaging. Radiol Med. 2012; 117: 96-101.

29. Lee JH, Kim HS, Yoon YC, Seo SW, Cha MJ, Jin W, et al. Characterization of small, deeply located soft-tissue tumors: Conventional magnetic resonance imaging features and apparent diffusion coefficient for differentiation between non-malignancy and malignancy. PLoS One. 2020; 15: e0232622.

30. Robba T, Chianca V, Albano D, Clementi V, Piana R, Linari A, et al. Diffusion-weighted imaging for the cellularity assessment and matrix characterization of soft tissue tumour. Radiol Med. 2017; 122: 871-9.

31. Choi YJ, Lee IS, Song YS, Kim JI, Choi K-U, Song JW. Diagnostic performance of diffusion-weighted (DWI) and dynamic contrast-enhanced (DCE) MRI for the differentiation of benign from malignant soft-tissue tumors. Journal of magnetic resonance imaging: JMRI. 2019; 50: 798-809.

32. Lee S-Y, Jee W-H, Jung J-Y, Park MY, Kim S-K, Jung C-K, et al. Differentiation of malignant from benign soft tissue tumours: use of additive qualitative and quantitative diffusion-weighted MR imaging to standard MR imaging at $3.0 \mathrm{~T}$. Eur Radiol. 2016; 26: 743-54.

33. Bonarelli C, Teixeira PAG, Hossu G, Meyer JB, Chen B, Gay F, et al. Impact of ROI positioning and lesion morphology on apparent diffusion coefficient analysis for the differentiation between benign and malignant nonfatty soft-tissue lesions. American Journal of Roentgenology. 2015; 205: W106-W13.

34. Dodin G, Salleron J, Jendoubi S, Abou Arab W, Sirveaux F, Blum A, et al. Added-value of advanced magnetic resonance imaging to conventional morphologic analysis for the differentiation between benign and malignant non-fatty soft-tissue tumors. Eur Radiol. 2020; 31:1436-1547.

35. Hassanien OA, Younes RL, Dawoud RM. Diffusion weighted MRI of soft tissue masses: Can measurement of ADC value help in the differentiation between benign and malignant lesions? Egyptian Journal of Radiology and Nuclear Medicine. 2018; 49: 681-8.

36. Del Grande $\mathrm{F}$, Ahlawat $\mathrm{S}$, Subhawong $\mathrm{T}$, Fayad LM. Characterization of indeterminate soft tissue masses referred for biopsy: What is the added value of contrast imaging at 3.0 tesla? J Magn Reson Imaging. 2017; 45: 390-400.

37. Teixeira PAG, Gay F, Chen B, Zins M, Sirveaux F, Felblinger J, et al. Diffusion-weighted magnetic resonance imaging for the initial characterization of non-fatty soft tissue tumors: correlation between T2 signal intensity and ADC values. Skeletal Radiol. 2016; 45: 263-71.

38. Song $Y$, Yoon YC, Chong $Y$, Seo SW, Choi YL, Sohn I, et al. Diagnostic performance of conventional MRI parameters and apparent diffusion coefficient values in differentiating between benign and malignant soft-tissue tumours. Clin Radiol. 2017; 72: 691.e1-.e10.

39. Jeon JY, Chung HW, Lee MH, Lee SH, Shin MJ. Usefulness of diffusionweighted MR imaging for differentiating between benign and malignant superficial soft tissue tumours and tumour-like lesions. Br J Radiol. 2016; 89: 20150929.

40. Lee SK, Jee W-H, Jung CK, Chung Y-G. Multiparametric quantitative analysis of tumor perfusion and diffusion with 3T MRI: differentiation between benign and malignant soft tissue tumors. The British journal of radiology. 2020; 93: 20191035.

41. Zhang X. The value of MRI intravoxel incoherent motion imaging (IVIM) and diffusion kurtosis imaging (DKI) in the differential diagnosis of benign and malignant bone and soft tissue tumors of lower extremity. Chin J Magn Reson Imaging. 2018; 9: 525-532.

42. Ahlawat S, Fayad LM. Diffusion weighted imaging demystified: the technique and potential clinical applications for soft tissue imaging. Skeletal Radiol. 2018; 47: 313-28

43. Surov A, Nagata S, Razek AA, Tirumani SH, Wienke A, Kahn T. Comparison of ADC values in different malignancies of the skeletal musculature: a multicentric analysis. Skeletal Radiol. 2015; 44: 995-1000.

44. Subhawong TK, Jacobs MA, Fayad LM. Diffusion-weighted MR imaging for characterizing musculoskeletal lesions. Radiographics. 2014; 34: 1163-77. 
45. Gimber LH, Chadaz TS, Flake W, Taljanovic MS. Advanced MR Imaging of Musculoskeletal Tumors: An Overview. Semin Roentgenol. 2019; 54: 149-61.

46. You MW, Yun SJ. Diagnostic performance of diffusion-weighted imaging for differentiating benign and malignant gallbladder lesions: A systematic review and meta-analysis. J Magn Reson Imaging. 2018; 48: 1375-88.

47. Padhani AR, Liu G, Koh DM, Chenevert TL, Thoeny HC, Takahara T, et al. Diffusion-weighted magnetic resonance imaging as a cancer biomarker: consensus and recommendations. Neoplasia. 2009; 11: 102-25.

48. Petscavage-Thomas JM, Walker EA, Logie CI, Clarke LE, Duryea DM, Murphey MD. Soft-tissue myxomatous lesions: review of salient imaging features with pathologic comparison. Radiographics : a review publication of the Radiological Society of North America, Inc. 2014; 34: 964-80.

49. Sigmund EE, Cho GY, Kim S, Finn M, Moccaldi M, Jensen JH, et al. Intravoxel incoherent motion imaging of tumor microenvironment in locally advanced breast cancer. Magn Reson Med. 2011; 65: 1437-47.

50. Bokacheva L, Kaplan JB, Giri DD, Patil S, Gnanasigamani M, Nyman CG, et al. Intravoxel incoherent motion diffusion-weighted MRI at 3.0 T differentiates malignant breast lesions from benign lesions and breast parenchyma. J Magn Reson Imaging. 2014; 40: 813-23.

51. Patel J, Sigmund EE, Rusinek H, Oei M, Babb JS, Taouli B. Diagnosis of cirrhosis with intravoxel incoherent motion diffusion MRI and dynamic contrast-enhanced MRI alone and in combination: preliminary experience. J Magn Reson Imaging. 2010; 31: 589-600.

52. Lewin M, Fartoux L, Vignaud A, Arrivé L, Menu Y, Rosmorduc O. The diffusion-weighted imaging perfusion fraction $\mathrm{f}$ is a potential marker of sorafenib treatment in advanced hepatocellular carcinoma: a pilot study. Eur Radiol. 2011; 21: 281-90.

53. Glenn GR, Tabesh A, Jensen JH. A simple noise correction scheme for diffusional kurtosis imaging. Magn Reson Imaging. 2015; 33: 124-33.

54. Bruno F, Arrigoni F, Mariani S, Splendiani A, Di Cesare E, Masciocchi C, et al. Advanced magnetic resonance imaging (MRI) of soft tissue tumors: techniques and applications. Radiol Med. 2019; 124: 243-52.

55. Kasprian G, Amann G, Panotopoulos J, Schmidt M, Dominkus M, Trattnig S, et al. Peripheral nerve tractography in soft tissue tumors: a preliminary 3-tesla diffusion tensor magnetic resonance imaging study. Muscle Nerve. 2015; 51: 338-45. 